\title{
Ocular Surface Squamous Neoplasia with Intraocular Extension: Clinical and Ultrasound Biomicroscopic Findings
}

\author{
Rachna Meel $^{\mathrm{a}}$ Rebika Dhiman $^{\mathrm{a}}$ Seema Sen $^{\mathrm{b}}$ Seema Kashyap ${ }^{\mathrm{b}}$ \\ Radhika Tandon ${ }^{\mathrm{a}}$ Murugesan Vanathi ${ }^{\mathrm{a}}$ \\ ${ }^{a}$ Oculoplasty and Ocular Oncology Services, Dr. R. P. Centre for Ophthalmic Sciences, All India Institute of \\ Medical Sciences, New Delhi, India; b Ocular Pathology Services, Dr. R. P. Centre for Ophthalmic Sciences, \\ All India Institute of Medical Sciences, New Delhi, India
}

\section{Keywords}

Ocular surface squamous neoplasia · Intraocular ocular surface squamous neoplasia - Ultrasound biomicroscopy

\section{Abstract}

Purpose: To study the clinical profile, ultrasound biomicroscopy (UBM) findings, and treatment outcome of ocular surface squamous neoplasia with intraocular extension (OSSNIO). Methods: It was a retrospective case series of 7 eyes of OSSN-IO treated at our center from March 2015 till December 2016. Results: Mean age of presentation was 53 years. Clinical signs of intraocular involvement were evident in 3 cases (43\%). In the remaining 4 cases (57\%), it was diagnosed on UBM alone. Mean tumor height on UBM was $5.06 \mathrm{~mm}(6.6$ $\mathrm{mm}$ for nodular and $3.8 \mathrm{~mm}$ for nodulo-ulcerative pattern). Of 7 cases, 2 were managed with exenteration, 4 cases with enucleation + limited excision \pm external beam radiotherapy, and 1 was lost to follow-up. Complete regression was noted in $83 \%$ cases (5/6) and they remain disease free at a mean follow-up of 23.2 months. One case died of metastasis at 7 months. Histopathological examination showed well- to moderately differentiated tumor in all cases. Conclusions:
Use of UBM in high-risk OSSN cases may help pick up IO invasion. Tumors that are at high risk for $\mathrm{IO}$ invasion are thick nodular tumors ( $>5 \mathrm{~mm}$ ), tumors with nodular or ulcerative morphology, and those with prior surgical intervention.

(c) 2018 S. Karger AG, Basel

\section{Introduction}

Intraocular invasion by ocular surface squamous neoplasia (OSSN-IO) is uncommon and is reported to occur in up to $13 \%$ cases [1-4]. It is seen in aggressive histopathological variants like spindle-cell, mucoepidermoid, and adenoid squamous OSSN [5-7]. In nonaggressive variety, previous history of surgical intervention is usually present [8]. Clinically, presence of anterior chamber cells, raised intraocular pressure, mass in the anterior chamber angle, and choroidal or exudative retinal detachment in an eye with OSSN is suggestive of IO invasion [1]. Recent advances in anterior segment imaging have refined our ability to accurately delineate the extent of tissue invasion, thus enabling early detection of IO tumor extension in the absence of any obvious clinical signs $[9,10]$, or

\section{KARGER}

(c) 2018 S. Karger AG, Basel 
where complete clinical evaluation is not possible like in tumors involving or overhanging the cornea or those with corneal opacity.

In this retrospective case series, we describe clinical, ultrasound biomicroscopic, and histopathological features of 7 cases of OSSN-IO, their management, and outcome.

\section{Materials and Methods}

A retrospective chart review was performed for all patients who were treated for OSSN-IO at our center from March 2015 till December 2016. The study was approved by the Institute's ethics committee and followed the tenets of the Declaration of Helsinki.

Demographic and clinical data including previous treatment history, risk factors, human immunodeficiency virus (HIV) status, systemic associations, best-corrected visual acuity, and laterality were noted. Clinical characteristics of tumor documented were location, size, height of tumor on ultrasound biomicroscopic examination (UBM), multiplicity, presence of feeder vessels, clinical appearance (gelatinous/pappiliform/ulcerative), growth pattern (nodular/sessile), presence of leukoplakia or pigmentation, tissue involved, and nodal and systemic metastasis. Clinical signs specifically noted to detect intraocular involvement were presence of anterior chamber cells, anterior chamber mass on gonioscopy, exudative retinal detachment, or choroidal mass on dilated fundus examination. UBM, ocular ultrasonography, and histopathological findings were documented in all cases. Imaging scans of orbit were reviewed in cases with forniceal involvement to identify orbital extension. Tumor was graded according to AJCC-TNM classification of conjunctival carcinoma (8th edition of the American Joint Commission on Cancer TNM Classification scheme Cancer (AJCC) Staging Manual [11]). Treatment advised, outcome, and follow-up was noted for patients, wherever available.

\section{Results}

A total of 57 eyes of 56 patients were treated for OSSN at our center during the study period. Out of these, 7 eyes $(12.3 \%)$ had intraocular extension. Mean age of presentation was $53 \pm 6$ years (range $35-80$ years). There was a marked male preponderance with male:female ratio of $6: 1$. Forty-three percent of patients $(3 / 7)$ were $\leq 50$ years at presentation. The mean duration from onset of symptoms to time of presentation was 7 months (range 2-12 months). The right eye was involved in 4 patients and the left eye in 2 patients. One case had bilateral OSSN (Case 3, Table 1) with intraocular extension in the left eye. Previous treatment history was present in 5/7 (71.4\%) cases and all 5 had undergone at least one previous surgery involving the tumor. One patient was HIV positive and 2 were treated for pulmonary tuberculosis.

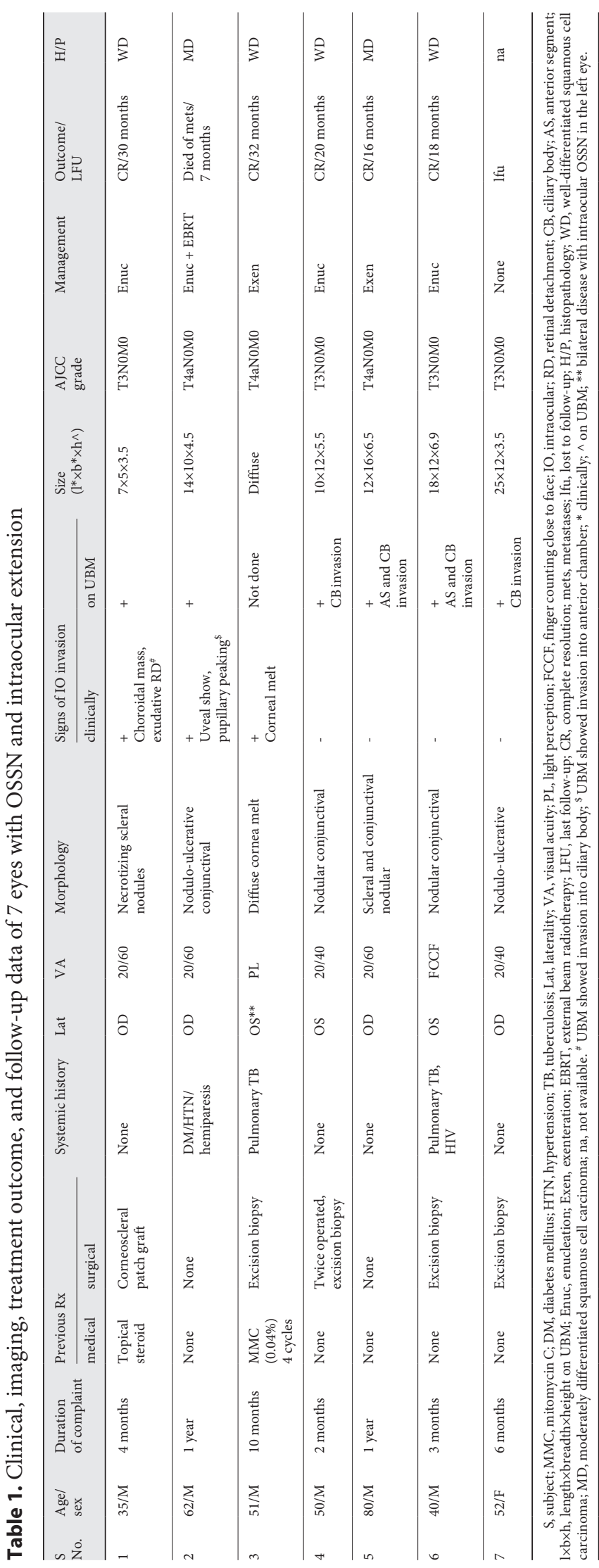



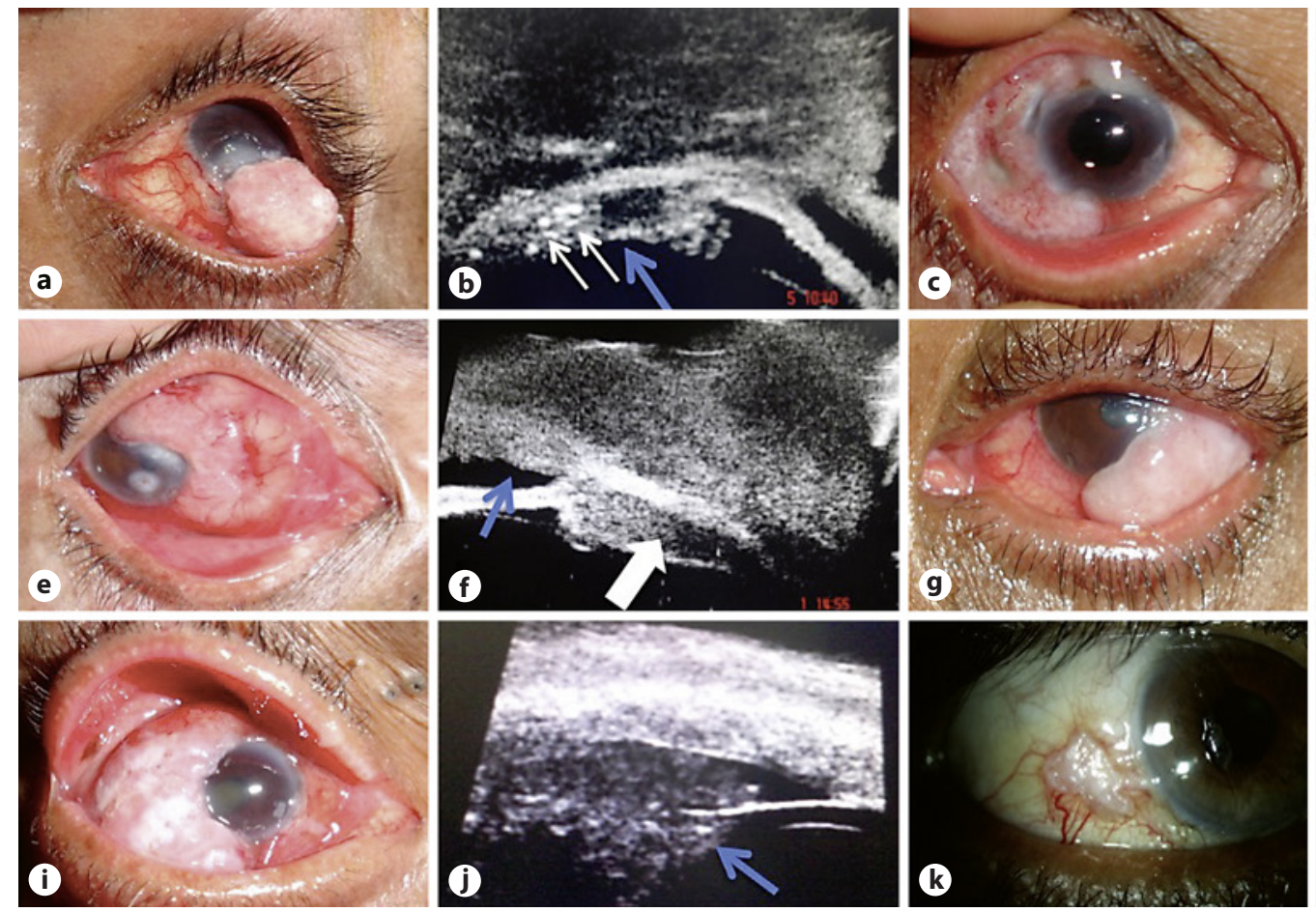
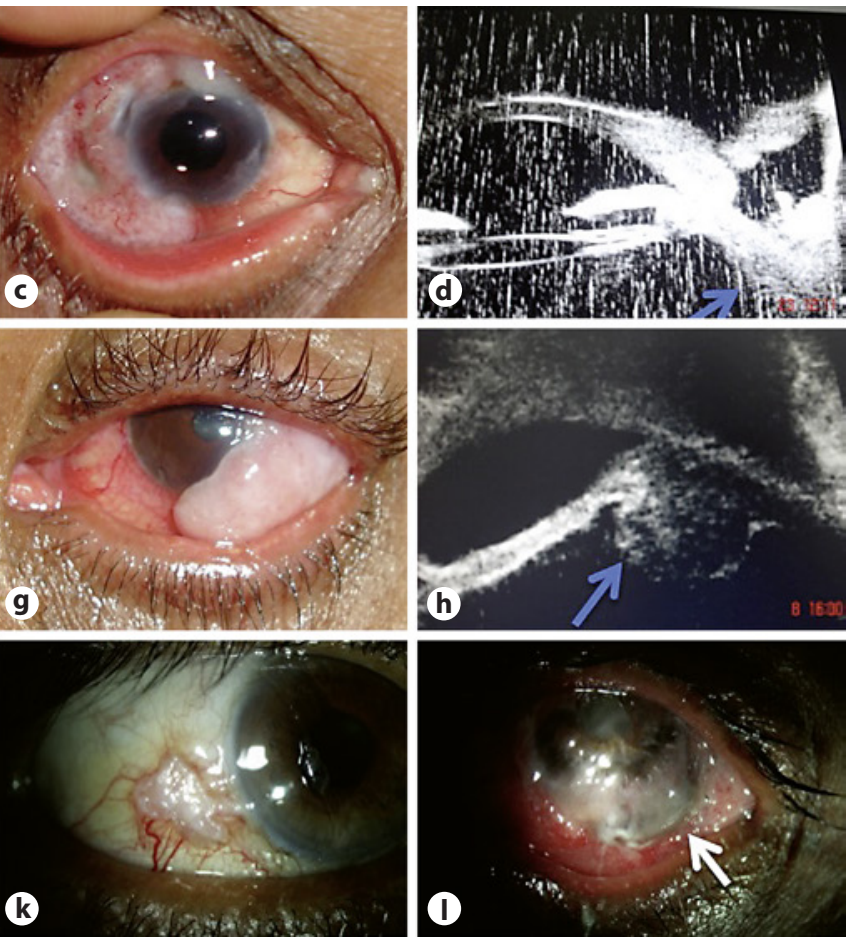

Fig. 1. a Clinical photograph showing a large nodular papillary OSSN of left eye in a young patient who was subsequently found to be HIV positive. $\mathbf{b}$ UBM images of the same case showing a thick tumor with invasion into ciliary body (blue arrow), areas of high echo-reflectivity possibly due to accumulation of keratin (small white arrows). c Clinical photograph showing a nodulo-ulcerative lesion in the right eye of a patient with previous history of surgical excision. d UBM image of the same patient showing ciliary body thickening (blue arrow). e Clinical photograph showing nodular OSSN of the right eye with subconjunctival nodules seen medially. f UBM images of the same case showing tumor inva- sion into anterior chamber (blue arrow) and ciliary body (white arrow). $\mathbf{g}$ Clinical photograph of a large nodular gelatinous OSSN. h UBM image of the same patient showing massive ciliary body invasion. i Nodular OSSN showing pupillary peaking - evidence of intraocular invasion. $\mathbf{j}$ UBM image of the same patient showing transcorneal invasion into the anterior chamber (blue arrow). $\mathbf{k}$, I Clinical photograph of the right and left eyes of the patient with bilateral OSSN. I Left eye (white arrow) showing diffuse OSSN with corneal melt and intraocular invasion as well as orbital extension; the patient had a history of tuberculosis. UBM was not possible in this case due to corneal melt.
Concomitant orbital extension was seen in $42.8 \%$ cases (3/7). The clinical, demographic, imaging, treatment, outcome, and follow-up data of the cases are summarized in Table 1.

The tumor morphology was nodular in 3 cases (Fig. 1a, e, h) and nodulo-ulcerative in 2 cases (Fig. 1d, k). Diffuse corneal melt possibly secondary to long-term mitomycin $\mathrm{C}$ (MMC $0.04 \%$ ) use was seen in 1 case (Fig. 11). One case presented with necrotizing scleral nodules and was misdiagnosed as nodular scleritis (Case 1, Table 1). Clinical signs of intraocular involvement were evident in 3 cases (43\%) as exudative retinal detachment with choroidal mass, pupillary peaking (Fig. 1k), and total corneal melt in one case each (Fig. 11). In the remaining 4 cases (57\%), intraocular involvement was diagnosed on UBM (Fig. 1c, f, i). Mean tumor height on UBM was $5.06 \mathrm{~mm}$ (range $3.5-6.9 \mathrm{~mm}$ ) for the cohort, $6.6 \mathrm{~mm}$ for nodular tumors, and $3.8 \mathrm{~mm}$ for nodulo-ulcerative tumors. UBM findings suggesting intraocular extension were ciliary body thickening in 4 cases, anterior segment invasion involving the anterior chamber angle in 2 cases, and transcorneal invasion into anterior segment in one case. Of these 4 cases, gonioscopy was not possible in 2 cases due to corneal opacity in one and due to overhanging tumor in another. In the remaining two cases, there were no obvious clinical signs of intraocular involvement. As per AJCC 8th edition classification for OSSN, 4 cases were staged as T3N0M0 and 3 cases as T4aN0M0. Figure 1 shows the clinical photographs and UBM imaging of patients of OSSN with intraocular invasion. 

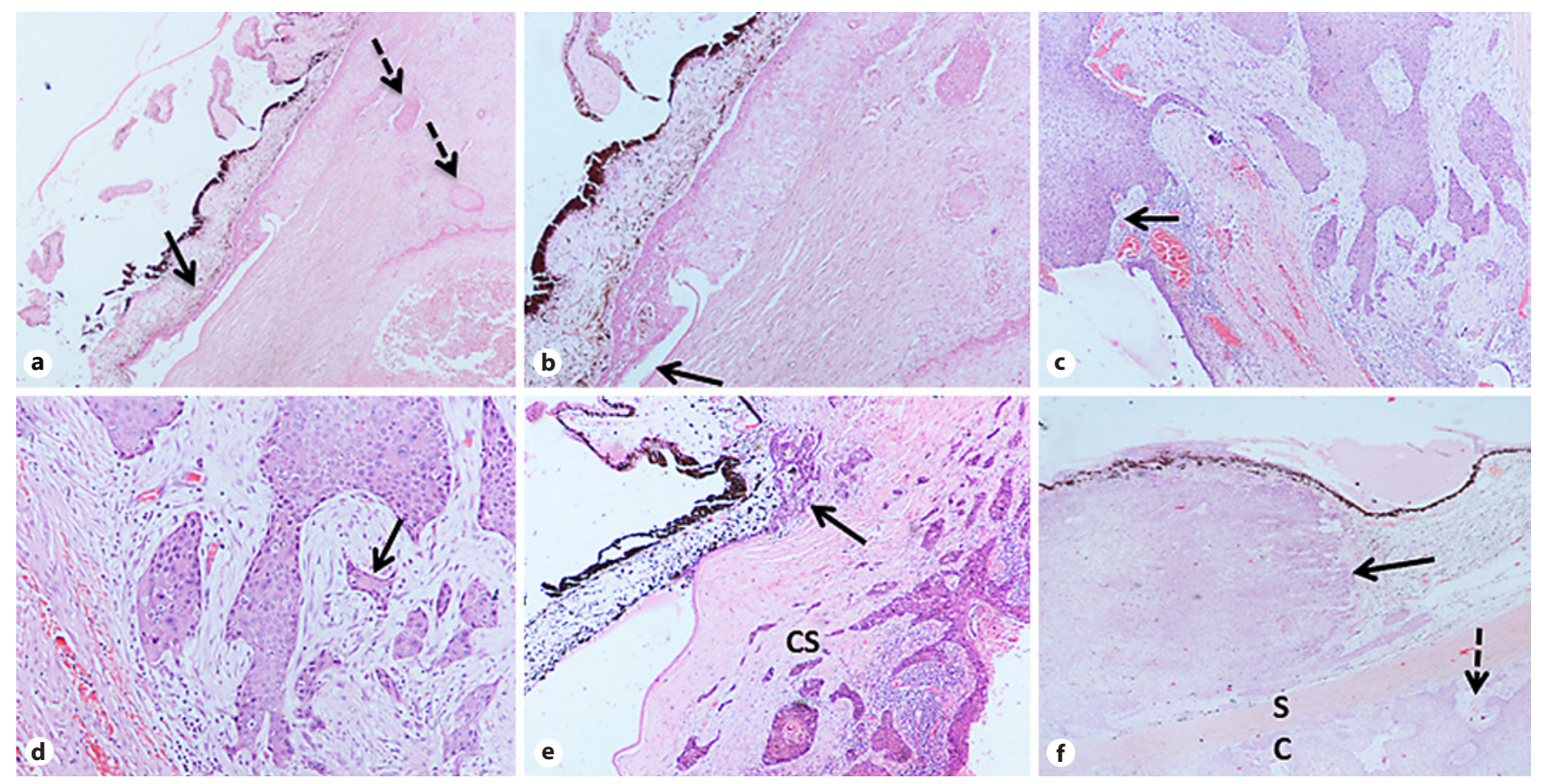

Fig. 2. Microphotograph $(\mathbf{a}, \mathrm{H} \& \mathrm{E}, \times 20)$ of invasive squamous cell carcinoma (SCC) infiltrating the anterior iris border (arrow) and adjoining sclera (dotted arrow). b H\&E $(\times 40)$ shows tumor infiltrating anterior chamber. Arrow points to the Descemet's membrane. c SCC arising from the limbus (arrow) and infiltrating episcleral tissues $(\mathrm{H} \& \mathrm{E}, \times 100)$. d H\&E $(\times 200)$ shows islands of infil- trating pleomorphic SCC cells with intracytoplasmickeratinization (arrow). e H\&E $(\times 40)$ of a SCC infiltrating the corneal stroma, iris root (arrow). f H\&E $(\times 20)$ shows SCC tumor infiltrating ciliary body stroma (arrow) and episcleral tissues (dotted arrow). CS, corneal stroma; SC, sclera.

\section{Treatment Outcome}

Of 7 cases, exenteration was carried out in 2 cases (AJCC T4) and enucleation with limited excision was performed in 4 cases ( 3 cases with AJCC T3 and 1 case with AJCC T4; adjuvant external beam radiotherapy [EBRT] was given to the case with AJCC T4 tumor). One patient was lost to follow-up after initial evaluation. Complete tumor regression was noted in $83 \%$ cases (5 out of 6 ) and they remain disease free with no tumor recurrence at a mean follow-up of 23.2 months (range 16-32 months). One case with AJCC T4 OSSN refused exenteration and therefore was managed with enucleation with limited excision followed by EBRT. Surgical margins were negative for tumor on histopathology. Ipsilateral preauricular lymph node metastasis was noted 4 months later and the patient was advised radical neck dissection. But the patient denied any further treatment and died of metastasis at 7 months of follow-up. Histopathological examination showed well- to moderately differentiated tumor in all cases. Figure 2 shows the histopathological features and sites of intraocular involvement in OSSN-IO. None of the cases had aggressive histopathological features consistent with spindle-cell, mucoepidermoid, or adenoid squamous OSSN. The surgical margins were negative and no local recurrence was seen in any case.

\section{Discussion}

OSSN-IO, although rare, is a potentially vision-threatening disease with high morbidity. Of all cases of OSSN managed at our center, $12.3 \%$ (7/57) eyes had IO involvement. This is comparable to $13 \%$ incidence reported in the literature [3]. In accordance with previous studies, there was a marked male preponderance in our series [3, $12]$. Mean age of patients in the current study was younger than reported in most other series [1, 4, 13-15].

In our series, obvious clinical signs of intraocular invasion were absent in $28.6 \%$ (2/7) of cases and could not be assessed with gonioscopy in another $28.6 \%(2 / 7)$ of cases due to a large tumor size covering the cornea or due to corneal opacity. At our center, we routinely perform 
UBM in cases of nodular OSSN, recurrent OSSN with history of surgical intervention, and in cases where gonioscopy is not possible. Signs of intraocular involvement on UBM are blunting of angles, anterior segment mass, and ciliary body thickening. Mean tumor height of OSSN on UBM as noted in the larger cohort of OSSN patients reported in an earlier study by authors was $2.93 \mathrm{~mm}$ [10]. This is lower than the mean tumor height of OSSN with intraocular invasion in the current series $(5.06 \mathrm{~mm})$. Also, in the current study, authors noted areas of high reflectivity on UBM in some tumors probably suggesting keratin accumulation within the tumor (Fig. 1b). This may be considered as a characteristic of OSSN on UBM, as is also the presence of keratinization clinically when compared with other ocular surface tumors.

OSSN-IO may mimic as necrotizing scleritis $[16,17]$, orbital cellulitis [18], sclerokeratitis [13], or pterygium [4]. These varied presentations often lead to delay in diagnosis and inappropriate management, thus predisposing to the spread of the tumor to deeper tissues. In our study, one case (Case 1, Table 1) was misdiagnosed as necrotizing scleritis and managed with a patch graft. A recurrence was noted shortly thereafter with IO spread of tumor, and an incision biopsy confirmed the diagnosis of OSSN. A recent study from the subcontinent has described an aggressive morphological pattern of OSSN, reported as the nodulo-ulcerative variant. It is reported to be associated with high incidence of intraocular (67\%) as well as orbital (33\%) extension [19]. In our study, two cases $(29 \%)$ presented with nodulo-ulcerative tumor morphology. It is to be noted that the mean height of tumors with nodulo-ulcerative morphology was less than the nodular variety. Also, subconjunctival scleral nodules were noted in one case in combination with conjunctival nodular tumor and this can be considered as an indication of intraocular invasion (Fig. 1).

Clinical risk factors reported to be associated with IO extension in OSSN are previous surgical intervention [1, $4,20]$, use of topical steroids $[17,20]$, and nodulo-ulcerative pattern of OSSN [18]. In addition, thick nodular tumors, perhaps more than $5 \mathrm{~mm}$, can also be taken as a risk factor for intraocular involvement, as seen in our study. We believe that nodulo-ulcerative tumors may be considered as having intraocular extension, as all cases with this morphology were found to have intraocular invasion in this study. In case UBM is not available, these must be treated like tumors with intraocular extension. In the current study, $71.4 \%$ cases had a previous history of surgery and one had a history of both surgery as well as steroid use (Case 1; Table 1). Tumor excision, unless done with proper technique, leads to high rate of recurrence that may be more aggressive and difficult to treat. The available surgical notes in the previously operated cases did not mention the use of cryotherapy or frozen section for control of surgical margins. We routinely perform enucleation in cases of OSSN-IO, and exenteration in cases with orbital extension (AJCC T4 disease). EBRT is given for AJCC T4 disease if surgical margins are positive for tumor on exenteration, or when enucleation with subtotal orbitectomy, that is, limited excision, is done (as in Case 2, Table 1).

Immunosuppression either post organ transplantation [21,22] or HIV [21, 23] infection have been reported to be associated with aggressive nature of the tumor, with a greater propensity for invasion of deeper structures. It is significant that $28.6 \%(2 / 7)$ patients had history of pulmonary tuberculosis in this series, and one of them (40 years old) had concurrent HIV infection, which was diagnosed on presentation (Case 6, Table 1). We wonder if this association is coincidental or indicates poor immunity in patients of HIV and tuberculosis, thus increasing the risk for invasive OSSN in the young patients.

Although histopathological variants like spindle-cell and mucoepidermoid carcinoma $[5,6]$ are known to be associated with risk of IO invasion, in our series all cases showed well- to moderately differentiated squamous cell carcinoma. We believe that the presence of risk factors can predispose to intraocular spread even in well-differentiated squamous cell carcinoma.

To conclude, clinically occult intraocular invasion in OSSN can be detected with judicious use of UBM in presence of high-risk factors like previous history of surgical intervention, immunosuppression, nodular or ulcerative tumor morphology, and a greater tumor thickness $(>5$ $\mathrm{mm}$ height). These clinical risk factors may be more commonly associated with intraocular invasion in OSSN than the unusual aggressive histopathology.

\section{Statement of Ethics}

The study was approved by the Institute's ethics committee and followed the tenets of the Declaration of Helsinki.

\section{Disclosure Statement}

No conflicting relationship exists for any author. 


\section{References}

1 Shields JA, Shields CL, Gunduz K, Eagle RC Jr: The 1998 Pan American Lecture. Intraocular invasion of conjunctival squamous cell carcinoma in five patients. Ophthal Plast Reconstr Surg 1999;15:153-160.

2 McKelvie PA, Daniell M, McNab A, Loughnan M, Santamaria JD: Squamous cell carcinoma of the conjunctiva: a series of 26 cases. Br J Ophthalmol 2002;86:168-173.

3 Tunc M, Char DH, Crawford B, Miller T: Intraepithelial and invasive squamous cell carcinoma of the conjunctiva: analysis of 60 cases. Br J Ophthalmol 1999;83:98-103.

4 Rootman DB, McGowan HD, Yücel YH, Pavlin CJ, Simpson ER: Intraocular extension of conjunctival invasive squamous cell carcinoma after pterygium surgery and cataract extraction. Eye Contact Lens 2012;38:133-136.

5 Tabbara KF, Kersten R, Daouk N, Blodi FC: Metastatic squamous cell carcinoma of the conjunctiva. Ophthalmology 1988;95:318321.

6 Cohen BH, GreenWR, Iliff NT, Taxy JB, Schwab LT, de la Cruz Z: Spindle cell carcinoma of the conjunctiva. Arch Ophthalmol 1980;98:1809-1813.

7 Mauriello JA Jr, Abdelsalam A, McLean IW: Adenoid squamous carcinoma of the conjunctiva: a clinicopathologic study of 14 cases. Br J Ophthalmol 1997;81:1001-1005.

8 Erie JC, Campbell RJ, Liesegang TJ: Conjunctival and corneal intraepithelial and invasive neoplasia. Ophthalmology 1986;93:176-183.
9 Thomas BJ, Galor A, Nanji AA, El Sayyad F, Wang J, Dubovy SR, Joag MG, Karp CL: Ultra high-resolution anterior segment optical coherence tomography in the diagnosis and management of ocular surface squamous neoplasia. Ocul Surf 2014;12:46-58.

10 Meel R, Dhiman R, Vanathi M, Pushker N, Tandon R, Devi S: Clinicodemographic profile and treatment outcome in patients of ocular surface squamous neoplasia. Indian J Ophthalmol 2017;65:936-941.

11 Conway RM, Graue GF, Pelayes DE, et al: Conjunctival carcinoma. In: Amin MB, Edge SB, Greene FL, et al, eds. AJCC Cancer Staging Manual 8th ed. Switzerland: Springer; 2017:787-793.

12 Shields CL, Demirci H, Karatza E, Shields JA: Clinical survey of 1643 melanocytic and nonmelanocytic conjunctival tumors. Ophthalmology 2004;111:1747-1754.

13 Mahmood MA, Al-Rajhi A, Riley F, Karcioglu ZA: Sclerokeratitis: an unusual presentation of squamous cell carcinoma of the conjunctiva. Ophthalmology 2001;108:553-558.

14 Nicholson DH, Herschler J: Intraocular extension of squamous cell carcinoma of the conjunctiva. Arch Ophthalmol 1977;95:843846.

15 Zhang Z, Li B, Shi J, Xu X, Li L, Gao F: Intraocular extension of conjunctival squamous cell carcinoma. Ophthalmologica 2007;221: 200-203.

16 Lindenmuth KA, Sugar A, Kincaid MC, Nelson CC, Comstock CP: Invasive squamous cell carcinoma of the conjunctiva presenting as necrotizing scleritis with scleral perforation and uveal prolapse. Surv Ophthalmol1988;33:50-54.
17 Sharma M, Sundar D, Vanathi M, Meel R, Kashyap S, Chawla R, Tandon R: Invasive ocular surface squamous neoplasia masquerading as nodular scleritis. Ophthal Plas Reconstr Surg 2017;33:e45-e47.

18 Kaliki S, Freitag SZ, Chodosh J: Nodulo-ulcerative ocular surface squamous neoplasia in 6 patients: a rare presentation. Cornea 2017; 36:322-326.

19 Rootman J, Roth AM, Crawford JB, Fox LP, Patel S: Extensive squamous cell carcinoma of the conjunctiva presenting as orbital cellulitis: the hermit syndrome. Can J Ophthalmol 1987;22:40-44

20 Searl SS, Krigstein HJ, Albert DM, Grove AS Jr: Invasive squamous cell carcinoma with intraocular mucoepidermoid features. Conjunctival carcinoma with intraocular invasion and diphasic morphology. Arch Ophthalmol 1982;100:109-111.

21 Shields CL, Ramasubramanian A, Mellen PL, Shields JL: Conjunctival squamous cell carcinoma arising in immunosuppressed patients (organ transplant, human immunodeficiency virus infection). Ophthalmology 2011;118: 2133-2137.

22 Kavoussi SC, Shetlar DJ, Barkmeier AJ, Carvounis PE: Invasive conjunctival squamous cell carcinoma presenting with serous retinal detachment. Can J Ophthalmol 2013;48: e158-e159.

23 Kamal S, Kaliki S, Mishra DK, Batra J, Naik $\mathrm{MN}$ : Ocular surface squamous neoplasia in 200 patients: a case-control study of immunosuppression resulting from human immunodeficiency virus versus immunocompetency. Ophthalmology 2015;122:1688-1694. 